\title{
Enhancing normal labour by adopting antenatal physiotherapy: a prospective study
}

\author{
Rooplekha Chauhan, Bharti Sahu*, Neetu Singh, Ritika Malviya, Priyadarshani Tiwari
}

Department of Obstetrics and Gynaecology, NSCB Medical College Hospital, Jabalpur - 482003, Madhya Pradesh, India

Received: 06 June 2016

Accepted: 01 July 2016

*Correspondence:

Dr. Bharti Sahu,

E-mail: arvindsahu15@yahoo.com

Copyright: $\odot$ the author(s), publisher and licensee Medip Academy. This is an open-access article distributed under the terms of the Creative Commons Attribution Non-Commercial License, which permits unrestricted non-commercial use, distribution, and reproduction in any medium, provided the original work is properly cited.

\begin{abstract}
Background: In the recent past there had been a steady rise in caesarean section rate as against the vaginal delivery. Information is still scarce on the benefits of physiotherapy and birthing experience of women who exercises in antenatal duration. Benefits of exercise during pregnancy include; prevention of excessive gestational weight gain, gestational diabetes, pre-eclampsia, decrease need for caesarean section and reduced low back pain. The objective of this study was to evaluation of antenatal physiotherapy on maternal and perinatal outcome and subjective evaluation of degree of labour pain perception.

Methods: In the study 150 primigravida subjects in second to third trimester without any associated high risk factor and were willing to exercise during pregnancy were chosen.

Results: In the study group, the average duration of latent phase [7.56 $( \pm 2.02)$ hours] and active phase of first stage of labour [3.26 $( \pm 1.06)$ hours] was significantly lesser as compared to the control group, $89.3 \%(\mathrm{n}=134)$ delivered vaginally and $10.7 \%(\mathrm{n}=16)$ subjects had to be taken for caesarean section. In the study group no perineal tear or need for any instrumentation (outlet forceps/ventouse) to facilitate delivery was seen. Higher proportion of the study group subjects i.e. $70.9 \%$ experienced moderate to mild pain during labour. and majority i.e. $72 \%$ subjects delivered newborns with birth weight $>2.5 \mathrm{~kg}$, with higher one minute and five minutes APGAR scores.

Conclusions: This highlights the very need for physiotherapy to be inculcated into the curriculum during antenatal period with conducive maternal and foetal outcome as it inflates the chances of normal deliveries and abate the rates of cesarean section and subsequent, which is the dire need of the day.
\end{abstract}

Keywords: Antenatal, Physiotherapy, Vaginal delivery, Caesarean section, Labour pain

\section{INTRODUCTION}

"It is from the womb of a woman that gives life to mankind into the world of light, a world in which to breathe and grow."

Women are primary care takers, first educators, bearers and nurtures of the next generation. Women's health is basic to women's advancement in all folds of endeavour. The pregnancy related disabilities result not only in human suffering but also effect social and economic empowerment. For maintaining health, exercise has been shown to improve health prospect in various ways, reduce body fat and overall weight, reduces blood pressure, and ensures better digestion, respiration and efficient circulation.

Routine exercise will enhance the health and reduce symptoms or complications (gestational diabetes, bladder and bowel problems, backache, fatigue, varicose veins) associated with pregnancy, labour and delivery. In addition, who maintains high level of fitness is more 
likely to quickly return to her pre-pregnancy health, figure and weight. While, exercise is a fundamental component of antenatal care, women's physical activity lessens, as the perception of risk during pregnancy is high. In the absence of medical or obstetrical complications, pregnant women are encouraged to continue and maintain active lifestyles during their pregnancies. ${ }^{1-3}$ Kegel's exercise is a commonly followed prenatal exercise used to strengthen pelvic floor muscle which may stretch during delivery, hence prepare for labour. $^{4-6}$ It is recommended that pregnant women can engage in moderate exercise for at least 30 minutes on most days of the week.

The incidence of caesarean sections is aiming skyward worldwide, particularly in India due to variety of reasons such as poor literacy, economic gains, patient response to adverse outcome, planning delivery on auspicious time, and greater safety for the baby, less pelvic floor trauma, avoidance of labour pains, rising number of previous sections and convenience. In 1985, World Health Organization in a consensus statement suggested that there may be no health benefits from caesarean section rates exceeding $10-15 \% .^{7}$ These figures have been however surpassed in most affluent societies and are now a subject of great public health concern.

\section{METHODS}

The design of this study was prospective case control study. Duration of this study was June 2011 to October 2012. Study was conducted department of obstetrics and gynaecology N.S.C.B. Medical College, Jabalpur, Madhya Pradesh, India.

The group in this study was primi gravid subjects without associated high risk factors from second to third trimester and willing were included in this study.

\section{Exclusion criteria}

Severe anemia, multiple gestation, heart disease, diabetes mellitus, muscloskeletal disorder, systemic infection, diastasis of recti, thyroid dysfunction, cervical incompetence, PIH/pre-eclampsia, vaginal bleeding, placenta praevia, contracted pelvis, oligohydraminos/ polyhydraminos, premature rupture of membranes, abnormal presentation.

Study group were chosen and complete protocol of physiotherapy exercises as per the trimester in which the subject got herself registered was explained to her and due verbal consent for enrollment in study was taken. They were taught the exercises with help of demonstration, videos and pictures and asked to practice the same at home and were evaluated on each subsequent visits to hospital. These cases were followed up in labour for

- Duration first and second stage
- Outcome of labour

- Pain as perceived by patient

- Perineal trauma if any

- Apgar score

The clearance of the ethical committee of Netaji Subhash Chandra Bose Medical College and Hospital, Jabalpur, was obtained before the commencement of the study.

\section{Exercises done during pregnancy}

Exercises done during pregnancy were kegel exercises, pelvic joint mobilization exercises. squatting, tailor press, pelvic rocking, bridging stretching exercises: calf stretching exercise, hamstring stretching exercise, quadriceps stretching exercise, adductor stretching exercise, gluteal stretching exercise breathing exercises diaphragmatic exercise, lateral costal breathing exercises, ventilatory muscle training exercises Ankle and foot exercises. Abdominal exercises: standing pelvic tilt, pelvis rocking exercises (cat and camel) posture correction exercises: these include standing lordosis correction exercises, standing- neck stretches, chin tuck exercise, standing- whole body stretch.

The study group cases were instructed to practice each set of exercises for at least 15-20 minutes daily at home. All the subjects were instructed to stop the exercises and report immediately to the emergency room in the case of bleeding, low back pain, abdominal contractions or gush of fluid.

\section{Statistical analysis}

The data was collected in a valid and reliable form through direct observation and analyzed, using the SPSS statistical software program (Version 11.5, Chicago, IL, USA). Chi-Square and T tests were used to compare the results and a $\mathrm{P}$ value of less than 0.05 was considered significant.

\section{RESULTS}

Table 1: Correlation between duration of latent and active phase of first stage labour in study and control groups.

\begin{tabular}{|llll||}
\hline \multicolumn{1}{|c|}{$1^{\text {st }}$ stage of $\quad$ Duration of labour (hours) } \\
labour & \multicolumn{1}{c|}{ Study group } & \multicolumn{1}{c|}{ Control } & \\
\hline Latent & $7.5664 \pm 2.02905$ & $9.7500 \pm 1.24298$ & $\mathrm{t}=5.302 ;$ \\
phase & $(\mathrm{n}=134)$ & $(\mathrm{n}=22)$ & $\mathrm{p}<0.0001$ \\
\hline $\begin{array}{l}\text { Active } \\
\text { phase }\end{array}$ & $3.2678 \pm 1.06190$ & $5.5577 \pm 1.18598$ & $\mathrm{t}=9.879 ;$ \\
\end{tabular}

The above table shows that in the study group $n=134$ underwent normal delivery with mean duration of latent phase of $7.56 \pm 2.029$ hours and active phase of $3.26 \pm 1.06$ hours. In the control group the mean duration of latent phase was $9.75 \pm 1.24$ hours and active phase of first stage of labour was of $5.55 \pm 1.18$ hours. Thus there was a 
significant decrease in the mean duration of both the phases of $1^{\text {st }}$ stage of labour in the study group as compared to the control group.

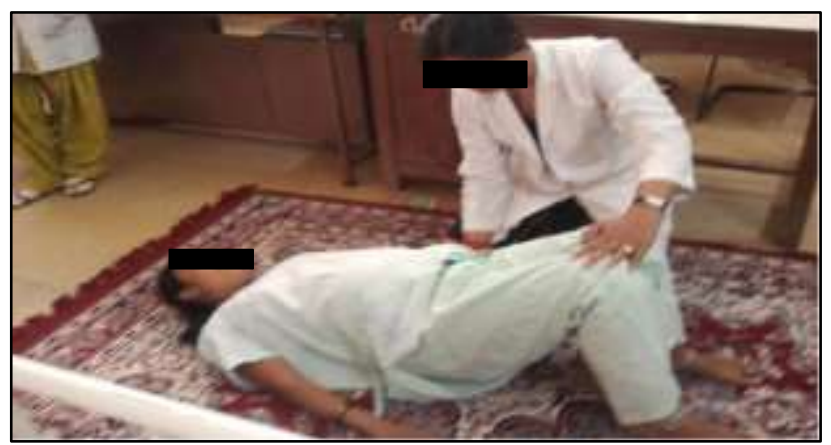

Figure 1: Exercise during 1st stage of labour.

Table 2: Correlation between duration of exercise (in weeks) and duration of latent phase and active phase of $1^{\text {st }}$ stage of labour.

\begin{tabular}{|lll|} 
Duration (weeks) & $\begin{array}{l}\text { Duration of } \\
\text { latent phase }\end{array}$ & $\begin{array}{l}\text { Duration of } \\
\text { active phase }\end{array}$ \\
\hline$\leq 10(\mathrm{n}=50)$ & $7.547 \pm 1.39$ & $3.20 \pm 10$ \\
\hline $10(\mathrm{n}=84)$ & $7.104 \pm 1.21$ & $2.82 \pm 0.92$ \\
\hline & $\mathrm{P}=0.0439$ & $\mathrm{P}=0.0257$ \\
\hline
\end{tabular}

Average duration of latent phase of 1st stage of labour in patients who did exercise $\leq 10$ weeks $(\mathrm{n}=50)$ was $7.547 \pm 1.39$ hours and in patients who did exercise $>10$ weeks $(\mathrm{n}=84)$ was $7.104 \pm 1.21$ hours which is statistically significant $(\mathrm{p}=0.0439)$.

Average duration of active phase of 1st stage of labour in patients who did exercise $\leq 10$ weeks $(\mathrm{n}=50)$ was $3.20 \pm 1.10$ hours and in patients who did exercise $>10$ weeks $(\mathrm{n}=84)$ was $2.82 \pm 0.92$ hours which is statistically significant $(\mathrm{p}=0.0257)$.

Table 3: Correlation between the mode of delivery in study and control group.

\begin{tabular}{|lll|}
\hline Mode of delivery & Study group (n) & Control (n) \\
\hline \multirow{2}{*}{ VD } & 134 & 22 \\
& $89.3 \%$ & $73.3 \%$ \\
\hline \multirow{2}{*}{ LSCS } & 16 & 8 \\
& $10.7 \%$ & $26.7 \%$ \\
\hline \multirow{2}{*}{ Total } & $\mathbf{1 5 0}$ & $\mathbf{3 0}$ \\
& $\mathbf{1 0 0 . 0 \%}$ & $\mathbf{1 0 0 . 0 \%}$ \\
\hline
\end{tabular}

$\chi^{2}=5.538 ; \mathrm{p}<0.01$.

The above table shows that $89.3 \%$ of the study group subjects underwent vaginal deliveries whereas only $73.3 \%$ of the controls underwent vaginal deliveries. The rest of the study group subjects underwent L.S.C.S. For indications of fetal distress. The above data is statistically significant showing that higher rates of vaginal deliveries are seen in the study group as compared to the control group.

Table 4: Correlation between presence of perineal tear in the vaginally delivered study and control group subjects.

\begin{tabular}{|lll|}
\hline Perineal tear & Study group (n) & Control group (n) \\
\hline- & 134 & 19 \\
& $100.0 \%$ & $86.36 \%$ \\
\hline+ & 0 & 3 \\
Total & $0.0 \%$ & $13.64 \%$ \\
\hline
\end{tabular}

The above table shows that there was no perineal tear in the study group delivered vaginally, whereas $n=3$ i.e. $13.64 \%$ of the controls delivered vaginally sustained a perineal tear. The above correlation is statistically significant showing lower rates of perineal tears in the study group who undertook physiotherapy exercise in the antenatal period.

Table 5: Correlation between the degree of pain perception in both study and control groups.

\begin{tabular}{|lll|}
\hline & Study group (n) & Control (n) \\
\hline \multirow{2}{*}{ Mild $<4$} & 30 & 0 \\
& $21.3 \%$ & $0.0 \%$ \\
\hline \multirow{2}{*}{ Moderate 5-7 } & 100 & 8 \\
& $70.9 \%$ & $28.6 \%$ \\
\hline \multirow{2}{*}{ Severe 8-10 } & 11 & 20 \\
& $7.8 \%$ & $71.4 \%$ \\
\hline \multirow{2}{*}{ Total } & $\mathbf{1 4 1}$ & $\mathbf{2 8}$ \\
\hline
\end{tabular}

$\chi^{2}=64.072 ; \mathrm{p}<0.001$

The above table describes the pain perception score in study group and control groups. ${ }^{8}$ In the study group $21.3 \%$ subjects experienced mild pain (score <4),70.9\% subjects experienced moderate pain(score 5-7) and 7.8\% subjects experienced severe pain (score 8-10). Similarly in the control group, $28.6 \%$ subjects experienced moderate pain (score 5-7) and $71.4 \%$ subjects experienced severe pain (score 8-10) and none experienced mild pain. Thus higher proportion of the study group $(70.9 \%)$ experienced moderate pain whereas higher proportion of the control group $(71.4 \%)$ experienced severe pain. This correlation is statistically significant. Thus it can be concluded that exercise in antenatal period helps alleviate pain during labour.

Table 6: Correlation between the APGAR SCORES at 1 minute and 5 minutes in study and control groups.

\begin{tabular}{|c|c|c|c|c|}
\hline \multicolumn{3}{|c|}{ APGAR at 1 minute } & \multicolumn{2}{|c|}{ APGAR at 5 minutes } \\
\hline & $<7$ & $>7$ & $<7$ & $>7$ \\
\hline $\begin{array}{l}\text { Study } \\
\text { group }\end{array}$ & $58(38.7 \%)$ & $\begin{array}{l}92 \\
(61.3 \%)\end{array}$ & $3(2 \%)$ & $\begin{array}{l}147 \\
(98 \%)\end{array}$ \\
\hline $\begin{array}{l}\text { Control } \\
\text { study }\end{array}$ & $18(60 \%)$ & $\begin{array}{l}12 \\
(40 \%)\end{array}$ & $\begin{array}{l}10 \\
(33.3 \%)\end{array}$ & $\begin{array}{l}20 \\
(66.7 \%)\end{array}$ \\
\hline
\end{tabular}


The above table shows that in the study group higher APGAR scores at 1 minute of $>7$ was observed in $n=92$ i.e. $61.3 \%$ subjects. In the control group only $n=12$ i.e. $40.0 \%$ subjects delivered new-borns with APGAR scores $>7$, the rest of the control $n=18$ i.e. $60.0 \%$ delivered new-borns with APGAR scores of $<7$. Thus statistically significant number of study group had higher APGAR scores as compared to the control groups.

The above table shows that the in study group higher APGAR scores at 5 minute of $>7$ was observed in $n=$ 147 i.e. $98.0 \%$ subjects. In the control group $n=20$ i.e. $66.7 \%$ subjects had APGAR scores $>7$, the rest of the control $\mathrm{n}=10$ i.e. $33.3 \%$ had APGAR scores of $<7$. Thus statistically significant number of study group subjects delivered new-borns with higher APGAR scores as compared to the control groups. This table thus shows a positive correlation between exercise and good perinatal outcomes.

\section{DISCUSSION}

The data on exercise during pregnancy are limited but suggest that moderate exercise during a low-risk pregnancy does not lead to adverse outcomes for the foetus or mother and improves overall maternal fitness and well-being.

\section{Duration of labour}

The results shows that the duration of latent phase of labour were $7.56 \pm 2.02$ and $9.75 \pm 1.24$ hours and active phase of labour $3.26 \pm 1.06$ and $5.55 \pm 1.18$ hours respectively for study and controls group indicating significant reductions $(\mathrm{P}<0.001)$. Average duration of latent phase of labour in patients who did exercise $\leq 10$ weeks $(n=50)$ was $7.547 \pm 1.39$ hours and in patients who did exercise $>10$ weeks $(n=84)$ was $7.104 \pm 1.21$ hours. Average duration of active stage of labour in patients who did exercise $\leq 10$ weeks $(n=50)$ was $3.20 \pm 1.10$ hours and in patients who did exercise $>10$ weeks $(n=84)$ was $2.82 \pm 0.92$ hours. Thus subjects who exercised for a longer duration in the antenatal period had shorter durations of both latent and active phases of labour. Salvesen $\mathrm{K}$ et al proposed that exercise may facilitate labour. In his study, 11 women randomized to pelvic floor muscle training had a lower rate of prolonged second stage labour than women allocated to no training, while Bell et al, in their study, agree that there is some evidence that weight-bearing exercises throughout pregnancy can reduce the length of labour and decrease delivery complications. ${ }^{9}$ Paisley et al, Sternfeld et al, Wolfe et al in their studies found that weight-bearing exercise throughout pregnancy can reduce the length of labour and decrease delivery complications. ${ }^{3,10,11}$

\section{Mode of delivery}

In our study $89.3 \%$ subject delivered vaginally and $10.7 \%$ subject had to be taken for caesarean sections for indication of fetal distress. In the control group $73.3 \%$ subject delivered vaginally and $26.7 \%$ subject were taken for caesarean section, indication being variable.

Studies in the past do not conclusively prove that exercise during pregnancy decreases the rates of primary caesarean sections. However our study reveals significant correlation between the two, thereby showing lower rates of primary caesarean sections and higher rates of vaginal deliveries in the exercising group of antenatal women.

\section{Perineal tears}

In the study group there were no perineal tears whereas $13.64 \%$ controls had perineal tears. Study of Sengul Donmez, Oya Kavlak also showed significant reduction in perineal trauma in women who practiced Kegels exercise antenatally. ${ }^{12}$

\section{Perception of degree of pain}

In the case group $21.3 \%$ subject's experienced mild pain, $70.9 \%$ subject's experienced moderate pain and $7.8 \%$ subjects experienced severe pain. Similarly in the control group, $28.6 \%$ subjects experienced moderate pain and $71.4 \%$ subjects experienced severe pain and none experienced mild pain.

Thus higher proportion of the case group i.e.70.9\% experienced moderate pain whereas higher proportion of the control group $71.4 \%$ experienced severe pain. Thus it can be concluded that exercise in antenatal period helps alleviate labour pains.

Exercise also may have a favorable effect on the subjective experience of discomfort during pregnancy. Sternfeld B et al reported that tracking exercise throughout pregnancy, women who exercised in the three months before pregnancy felt better during the first trimester than those who did not exercise. ${ }^{10}$ Exercise in the first and second trimesters was correlated with feeling better in the third trimester.

\section{Apgar scores at 1 and 5 minutes}

In our study statistically significant number of subjects had higher APGAR scores at 1 and 5 minutes as compared to the control groups thus showing a positive correlation between exercise and good perinatal outcome.

In the study by Kardel, Botkin and Driscoll and Lokey there was no significant difference in APGAR scores at birth in the neonates of exercising and non- exercising mothers. ${ }^{13-15}$ They also reported that the first and fifth minute APGAR scores of neonates of exercising and nonexercising mothers did not differ. Also in our study the newborns of the physiotherapy group of women had lesser rates of intubations $2.0 \%$ and nursery admissions $6.7 \%$ as compared to the control group i.e. $16.7 \%$ and $20.0 \%$ respectively. 
For generalizing the result study should be conducted in future on a sample size representing the target population. Our limitation was exclusion of females with multiple parity.

\section{CONCLUSION}

A review of the evidence suggests that, in most cases, exercise is safe and beneficial for both mother and foetus during pregnancy and women should therefore be encouraged to commence and endure exercise to derive the health benefits. Antenatal physiotherapy, by reducing the rates of primary caesarean sections, the rates of caesarean sections for previous section also curtails and the associated morbidity and mortality due to conditions like uterine dehiscence, uterine rupture, hysterectomy, placenta praevia, thrombo-embolic diseases, uterine infections and longer hospital stays dwindles. There is some affirmation to suggest that physiotherapy is compelling in mitigating high pain levels associated with positional difficulties and may facilitate spontaneous rotation. Despite the substantial health benefit of exercise during pregnancy, rates of exercise among pregnant women are surprisingly low compared with the general population. Practitioners should encourage exercise among healthy pregnant women by addressing the unique barriers to exercise experienced by pregnant women (eg, nausea, fatigue, out-dated advice that pregnant women should refrain from exercise). Promoting exercise during pregnancy needs to remain a crucial objective among health promoters. However, even among the general population, engaging in regular exercise is a complex and challenging behaviour. Being pregnant presents further challenges to an already difficult behaviour. Albeit, there are many opportunities for future research and continuous efforts to study exercise during pregnancy will increase our knowledge about the determinants and outcomes of exercise participation and improve our ability to effectively intervene.

\section{Funding: No funding sources}

Conflict of interest: None declared

Ethical approval: The study was approved by the Institutional Ethics Committee

\section{REFERENCES}

1. Mottola, MF, McLaughlin R. Exercise and pregnancy: Canadian guidelines for health care professionals. Wellspring. 2011;22(4):A1-4.
2. Zavorsky GS, Longo LD. Exercise guidelines in pregnancy. Sports Medicine. 2011;41(5):345-60.

3. Paisley TS, Joy EA, Price RJ. Exercise during pregnancy: a practical approach. Curr Sports Med Rep. 2003;2:325-30.

4. Park SH, Kang CB, Jang SY, Kim BY. Effect of kegel exercise to prevent urinary and fecal incontinence in antenatal and postnatal women: systematic review. Journal of Korean Academy of Nursing. 2013;43:420-30.

5. Hamid El, Azzam AA, Ismail HF, Gaafar HM. Effect of a structured antenatal kegel exercises protocol on labor progress among women attending antenatal clinics. Egyptian Nursing Journal. 2012;3:2090-210.

6. Dumoulin C, Hay-Smith J. Pelvic floor muscle training versus no treatment, or inactive control treatments, for urinary incontinence in women. Cochrane Database Syst Rev. 2010;(1):CD005654.

7. World Health Organization. Appropriate technology for birth. Lancet. 1985;2:(8452):436-7.

8. Bieri D, Reeve RA, Champion GD, Addicoat I. The Faces Pain Scale for the self-assessment of the severity of pain experienced by children: development, initial validation, and preliminary investigation for ratio scale properties. Zeigler. 1990;41(2):139-50.

9. Bell BB, Dooley MMP. Exercise in pregnancy. guidelines and audit committee of the RCOG Statement No. 4; 2006.

10. Sternfeld B. Physical activity and pregnancy outcome: review and recommendations. Sports Med. 1997;23:33-47.

11. Wolfe LA, Hall P, Webb KA, Goodman L, Monga M, McGrath MJ. Prescription of aerobic exercise during pregnancy. Sports Med.1989;8:273-301.

12. Dönmez S, Kavlak O. Effects of prenatal perineal massage and kegel exercises on the integrity of postnatal perine. Health. 2015;7:495-505.

13. Kardel KR, Kase T. Training in pregnant women: effect on fetal development and birth. Am J Obstet Gynecol. 1998;178(2):280-6.

14. Botkin C, Driscoll CE. Maternal aerobic exercise: newborn effects. Fam Pract Res J. 1991;11(4):38793.

15. Lokey EA, Tran ZV, Wells CL, Myers BC, Tran AC. Effects of physical exercise on pregnancy outcomes: a meta-analytic review. Med SciSports Exerc. 1991;23(11):1234-9.

Cite this article as: Chauhan R, Sahu B, Singh N, Malviya R, Tiwari P. Enhancing normal labour by adopting antenatal physiotherapy: a prospective study. Int J Reprod Contracept Obstet Gynecol 2016;5:2672-6. 